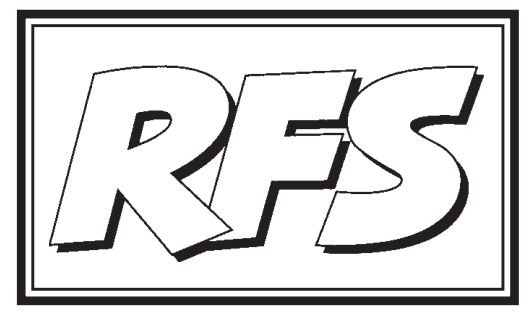

Revista de Fomento Social, 59 (2004), 177-193

\title{
Notas para un debate sobre el Foro Social Mundial
}

\section{Chico WHITAKER ${ }^{1}$}

(PALABRAS ClaVE: Foro SOCIAL MUNDIAL, Foro ESPACIO, CREACIÓN DE REDES. KEY WORDS: WORLD SOCIAL FORUM, FORUM-SPACE, INTER-LINKING)

En relación con la posibilidad de éxito del Foro Social Mundial y su proceso de mundialización surgen muchas cuestiones sobre su continuidad. Varias valoraciones han sido escritas en diferentes direcciones, así como surgen nuevas propuestas sobre la organización. Sin duda, el Foro enfrenta una crisis positiva, de crecimiento, que exige la profundización de algunas cuestiones suscitadas por su Carta de Principios. Para no correr el riesgo de destruir sus potencialidades, se hace necesario y urgente superar algunas ambigüedades, antes que el avance del proceso cristalice en orientaciones

1 El autor, brasileño, es uno de los fundadores del Foro Social Mundial. Agradecemos al profesor Rafael Díaz Salazar su gentileza al proporcionarnos el presente texto. Traducción del original portugués de Adolfo Rodero Franganillo. 
sin retorno. El presente texto pretende contribuir a ese debate, abordando tres cuestiones que son básicas para la continuidad del Foro:

- La opción entre Foro espacio y Foro movimiento.

- La importancia relativa, en los Foros, de las actividades autoorganizadas por los participantes y las actividades programadas por el Comité Organizador y el carácter de estos dos tipos de actividades.

- El papel de los Comités Organizadores en los eventos del Foro.

La primera de las cuestiones es la más decisiva, porque de la opción adoptada se deducen respuestas diferentes para las demás. Una cuarta cuestión que debería ser abordada es la relación con los partidos políticos. En estas notas me voy a detener solamente en las tres cuestiones indicadas.

\section{Foro: ¿espacio o movimiento?}

Considerar el Foro como un espacio o considerarlo como un movimiento es una opción básica y preliminar en la etapa del proceso en que estamos entrando. Escamotear una respuesta o no establecerla con claridad es la mejor manera de crear dificultades.

En la Carta de Principios del Foro se define perentoriamente como un espacio. Pero no todos piensan o actúan como si realmente fuese solamente un espacio, o por lo menos como si debiese permanecer siempre como un espacio.

Para muchos es un espacio que tiene algo de un movimiento. Para otros, es "todavía" solamente un espacio. Es decir, el Foro puede y debe transformarse en un enorme movimiento, o en un movimiento de movimientos, como algunos periodistas llegan a llamarlo. El estruendoso éxito de las manifestaciones del 15 de febrero de 2003 en todo el mundo contra la guerra -que llevó a los más entusiastas a considerar que este hecho afecta también al Foro, llegando a decir que fue directamente el Foro quien lo promovió...- estimula todavía más el deseo de que el Foro asuma el papel fundamentalmente movilizador que tienen los movimientos.

Ahora bien, movimientos o espacios son cosas completamente diferentes. Sin maniqueísmos simplificadores, o se es una cosa o se es otra. No son excluyentes, esto es, pueden coexistir. Tampoco son antagónicos, esto es, no se neutralizan mutuamente, y pueden también sumarse. Pero no se puede 
ser las dos cosas al mismo tiempo, no se puede ser un poco de cada una -lo que acabará perjudicando una a otra. Movimientos o espacios pueden estar dirigidos, cada uno según su papel, a objetivos comunes sobre todo de carácter general. Pero cada uno funciona de una forma que le es propia, sirviendo a objetivos específicos diferentes.

Una verdadera cuestión a planearnos es la siguiente: transformar el Foro Social Mundial en un movimiento, ahora - o no ahora, sino más adelante durante el progreso del proceso- ¿es una buena estrategia para alcanzar el objetivo de todos los participantes, es decir, la superación del neoliberalismo y la construcción de "otro mundo posible"? O, en sentido inverso, ¿es útil, para alcanzar ese objetivo, que podamos seguir contando -ahora o a lo largo del desarrollo del proceso- de espacios como los que ha abierto el Foro Social Mundial?

Por mi parte, no tengo dudas de que es fundamental asegurar a cualquier precio la continuidad del Foro en cuanto espacio y no ceder a ninguna tentación de transformarlo ahora -o más adelante- en un movimiento. Si lo mantenemos como espacio, ello no impedirá ni perjudicará -antes bien, al contrario- la formación y el desarrollo de muchos movimientos. Pero si optamos por transformarlo en un movimiento, dejará de ser un espacio, perdiendo todas las potencialidades que tienen los espacios.

Más aún: si se hace así, nosotros mismos estaremos -sin necesitar de la ayuda de los que combatimos- deteriorando un poderoso instrumento de lucha que hemos sido capaces de crear a partir del más precioso descubrimiento de los últimos tiempos: una fuerza libre de articulación horizontal, que explica el éxito tanto de Porto Alegre como de Seattle y de las manifestaciones del 15 de febrero contra la guerra. Y no podemos dejar de considerar que una articulación social horizontal todavía tiene mucho que contribuir a nuestra lucha actual, ella sería necesaria también para el propio proceso de construcción del mundo que queremos.

Esta certeza se fundamenta en el análisis de las ventajas del carácter actual del Foro como espacio frente a una eventual condición de Foro-movimiento. 


\section{En qué se diferencian movimientos y espacios}

Un movimiento congrega personas -sus militantes, así como los militantes de los partidos- que deciden organizarse para realizar, colectivamente, determinados objetivos. Su formación y existencia implican por tanto una definición de estrategias para alcanzar esos objetivos, la formulación de programas de acción y la distribución de responsabilidades entre sus miembros -entre otras, las de la dirección del movimiento. Los que asumen esa función liderarán a los militantes del movimiento, llevándolos -con autoritarismo o con métodos democráticos, según la elección que hayan hecho los creadores del movimiento- a cumplir la parte que corresponde a cada uno en la acción colectiva. Su estructura organizativa es, por tanto, piramidal, necesariamente, por más democrático o participativo que sea el proceso interno de decisiones en el movimiento y la manera de escoger a los que ocuparán los diferentes niveles de dirección que comporta siempre una pirámide. y su eficacia dependerá de la claridad y de la precisión de sus objetivos específicos y, por tanto, de sus propias limitaciones en el tiempo y en el espacio.

Un espacio no tiene líderes. Es sólo un lugar, fundamentalmente horizontal, tal como una extensión de la tierra, puesto que puede comprender altos y bajos. Es como una plaza sin dueño -si ésta tiene un dueño que no admite a la colectividad deja de ser una plaza y pasa a ser un terreno particular. Unas plazas son espacios abiertos en general que pueden ser usados por todos los que encuentran algún tipo de interés en utilizarlos. No tienen otro objetivo salvo el de ser plazas, prestando el servicio que necesitan los que las utilizan. Cuanto más tiempo permanezcan como plazas, mejor para aquellos que aprovechan lo que ofrecen para la realización de sus respectivos objetivos.

Por otro lado, aunque una plaza tiene árboles y pequeñas colinas en su interior, se trata de un espacio socialmente horizontal. Quien sube a los árboles o a las colinas no puede pretender, desde lo alto, liderar todo o parte de lo que hagan los que se encuentran en la plaz1. Lo mínimo que les puede suceder es ser ridiculizados por aquellos a los que pretenden liderar. O, si fueran muy insistentes e incómodos, acabarían hablando solos, forzados por los demás frecuentadores de la plaza a abandonarla, pudiendo incluso volver posteriormente con "autoridades públicas" que les hagan salir o parar de arengar desde sus árboles o colinas, si se estima que están perturbando la tranquilidad que debe caracterizar las plazas públicas. 


\section{El Foro como espacio incubador de movimientos}

La Carta de Principios del Foro recoge claramente su contraposición al establecimiento de cualquier tipo de dirección o de liderazgo dentro de aquél: nadie puede hablar en nombre del Foro -no cabe hablar en nombre de un espacio- ni de sus participantes. Todos -personas y organizaciones- conservan su derecho de expresarse o de actuar durante o posteriormente a su celebración según sus convicciones, asumiendo o no posiciones y propuestas que tengan o que sean presentados por otros participantes, pero nunca en nombre del Foro o del conjunto de sus participantes.

Como en las plazas, el Foro es un espacio abierto, como también especifica la Carta de Principios. Pero no es un espacio neutro, como en las plazas públicas. Se abre de tiempo en tiempo y en diferentes lugares del mundo-durante los eventos que lo concretan- con un objetivo específico: permitir que el máximo posible de personas, organizaciones y movimientos que se oponen al neoliberalismo puedan encontrarse libremente, escucharse unos a otros, aprender con las experiencias y luchas de los demás, discutir propuestas de acción, articular nuevas redes y organizaciones que traten de superar el actual proceso de globalización dominado por las grandes corporaciones internacionales y por los intereses financieros. Es, por tanto, una espacio creado para servir a un objetivo común de todos los que acuden a él, que funciona horizontalmente como una plaza pública, sin líderes ni pirámides de poder en su interior. Todos los que acuden al Foro se disponen a aceptar esa perspectiva -por eso mismo se establece que para entrar en la "plaza" es preciso estar de acuerdo con su Carta de Principios.

De hecho, el Foro funciona como una "fábrica de ideas", o una incubadora, de la cual se espera que surja el máximo posible de nuevas iniciativas dirigidas a la construcción de otro mundo, que todos consideran posible, necesario y urgente. De cualquier modo, se espera que de aquel nazcan más y más movimientos, mayores o menores, más o menos combativos, cada uno con sus objetivos específicos, para cumplir sus papeles en la misma lucha para cuyo desarrollo fue abierta la plaza.

Verdaderamente, la mayor potencialidad del Foro-espacio es exactamente esta: hacer surgir movimientos que amplíen una lucha. Cuando de un movimiento nacen nuevos movimientos, estos surgen en oposición a aquél, como resultado de sus divisiones internas. Y esto es lo que sucedería si el Foro se transformase en un movimiento. 
Los objetivos de estas nuevas iniciativas, a su vez, no precisan ser todos claros y precisos, a diferencia de lo que ocurre en los movimientos. Algunos pueden incluso estar siendo todavía intuidos -fomentados en la incubadora- exigiendo tiempo para su maduración.

Por otro lado, se acepta en el Foro que cada uno se empeñe con su mayor o menor fervor en la lucha común, según la etapa en que se encuentre en su propio camino de compromiso con la lucha de la humanidad por otro mundo. Mientras que en un movimiento hay un compromiso mutuo entre sus participantes.

\section{La ventaja de no tener un "documento final"}

La Carta de Principios del Foro refuerza todavía más esa perspectiva al tratar la cuestión de eventuales "documentos finales". Aunque se consiguiese que no fuesen reductores o simplificadores, como ocurre en general con los "documentos finales", el Foro no los tiene, en cuanto Foro. No se trata de una opción para no comprometerse con una lucha y con una movilización necesaria para el enfrentamiento con el neoliberalismo, como pueden querer interpretar los más preocupados en transformar el Foro en un movimiento. Lo que ocurre es que una plaza no hace "declaraciones". Es evidente que aquellos que están presentes en ella pueden hacerlas. Los participantes en el Foro Social Mundial pueden hacer todas las declaraciones finales que quieran, y es bueno que las hagan. Pero nunca serán declaraciones del Foro en cuanto Foro. Como espacio común a todos, el Foro no "habla". O, en otro sentido, "habla" y mucho", pero según su propia existencia. En la medida en que cada vez más gentes y organizaciones se unen para encontrar caminos de superación del neoliberalismo, éste es un hecho político muy expresivo. No es necesario que nadie se ponga a hablar en su nombre.

Todos y cada uno de los documentos o declaraciones de propuestas serán así una manifestación del que o de los que la suscriben libremente, sin presiones ni controles de tomas de posición. Por ello mismo, el Foro establece que las declaraciones o propuestas no pueden ser votadas o aclamadas por los participantes del Foro, en cuanto manifestaciones del conjunto de los que frecuentan la "plaza". Verdaderamente, esto haría que muchos se apartaran del Foro-espacio, por no aceptar o no estar de acuerdo con los líderes que estuviesen pretendiendo, de forma ridícula, conducirlos a lo alto de sus árboles o colinas. 
Esta opción adoptada por el Foro fue además bien comprendida por el gran número de participantes que asistieron en la última reunión del Foro en Porto Alegre, al "Mural de Propuestas de acción durante el Foro de 2003". En aquel lugar se abrió un espacio para que todos se expresaran; las propuestas o declaraciones finales presentadas en ese Mural -o enviadas a él posteriormente- dan cuenta precisamente de la riqueza y diversidad de los compromisos de los participantes. Se encuentran a disposición de todos los interesados en la propia página del Foro, pero no se consiguió este año hacer visible todo los que sus participantes decidieron a partir del Foro, puesto que el Mural, como innovación introducida en su edificio, fue insuficientemente divulgado.

Su actual divulgación a través de Internet con una indicación sobre cómo contactar con los autores de las propuestas- ya abre, sin embargo, otras perspectivas: ello permitirá que las articulaciones surgidas en torno a estas propuestas durante el Foro se amplíen ahora todavía más, a través de nuevos contactos y relaciones que pasan a ser posibles. Como si una "plaza" del Foro pasase a permanecer permanentemente abierta, extendiéndose temporal y espacialmente afuera, mucho más ampliamente del tiempo del espacio limitado a los cinco días de Porto Alegre. Apoyándose ahora en las enormes posibilidades abiertas por Internet, pueden transformarse en más contactos y más acciones concretas. Lo mismo puede suceder con "Murales de propuestas" que sean montados en otros eventos.

Pero el Foro-espacio tiene todavía otras ventajas.

\section{La diversidad}

Como espacio abierto, el Foro tiene la posibilidad de asegurar el respeto a la diversidad, en contraste con lo que ocurriría si fuera un movimiento. El principio del respeto a la diversidad, adoptado en la Carta de Principios, tiene verdaderamente una importancia más profunda: está fundado en la certeza de que una de las características fundamentales de ese otro mundo que pretendemos construir -como también decimos ya, de "otros mundos posibles"- debe ser precisamente el respeto a la diversidad.

Como resultado de este principio el Foro también permite -sin caer en la absoluta neutralidad de las plazas públicas- que cada uno conserve su libertad de escoger el sector o el nivel en el que actúan para transformar una realidad. Esa actuación puede ser tanto amplísima e incluyente como bastante 
restringida; puede pretender influir tanto en las causas más profundas de los problemas que enfrenta el mundo, como en los efectos meramente superficiales de esos problemas. La gama de temas discutidos durante el Foro y de los objetivos perseguidos en él puede ser, de esta manera, bastante amplia, como ocurre con la gama de mudanzas que la construcción de un nuevo mundo exige. Nadie dentro del Foro tiene el poder, o el derecho de decir que esta o aquella acción o propuesta es más importante que otra o quizás que las demás, ni, por tanto, el poder o el derecho de dar o defender una mayor visibilidad a sus propias propuestas, "usurpando" para sus propios objetivos o espacio lo que es de todos.

Esto, además, es una cuestión sobre la que se debería reflexionar con cuidado, frente a lo que viene ocurriendo con las "marchas" o manifestaciones callejeras con las que los foros tienden a terminar. Sus banderas deberían ser banderas de todos, como expresión visible de su diversidad y de la variedad de propuestas que en él se contienen o de él nacen. Privilegiar esta u otra bandera, en las "comisiones" de encabezamiento o en la designación de eventuales oradores en los actos finales de las marchas, es contradictorio con el principio de respeto de la diversidad y representa una visión de Foro-movimiento y no de Foro-espacio. Pero esta es otra cuestión a discutir.

Ahora bien, todas estas características del Foro explican ciertamente su gran aceptación y el poder de atracción y el éxito de sus eventos. Sus participantes sienten que sus opciones son respetadas en cuanto al ritmo y en la profundidad de sus compromisos. Hay los que pueden ver el Foro como militantes pero la mayor parte de sus participantes no lo hacen por obligación o por obediencia a las órdenes de los jefes. Acuden al Foro por su propia convicción de que es importante venir, para intercambiar experiencias, aprender a articularse, manteniendo la libertad que tenían antes y que continuarán teniendo durante y después de su participación en el evento. Saben que en él no recibirán órdenes ni tendrán que seguir palabras de orden, que no serán pagados, por lo que no tendrán que rendir cuentas de lo que hicieron o no hicieron, que no tendrán que dar pruebas de fidelidad y disciplina, no serán expulsados si no lo hicieron -todo lo contrario de lo que les ocurriría si hubiesen ido a participar en algún encuentro de un colectivo organizado. 


\section{La alegría y la corresponsabilidad}

Yo también afirmaría que ese carácter del Foro explica la gran alegría que reina en esta "plaza", como en una enorme feria -una verdadera fiesta con espacio inclusive para manifestaciones y actuaciones de diferentes tipos en los espacios de tránsito. Nadie se angustia porque nadie tiene que luchar para que sus ideas y propuestas prevalezcan sobre las demás. Nadie está preocupado por defenderse de otros que pretendan controlarlo, imponer orientaciones o reglas de comportamiento-menos aún de comportamientos políticos, como en las "bancadas" o "delegaciones" que tienen que reunirse para valorar, decidir, asumir tarifas, como en los buenos y disciplinados partidos o movimientos. Tales reuniones son posibles incluso, pero nunca obligatorias para quien no sea militante de éste o de aquel movimiento. Aquellos que quieran aprovechar la oportunidad para hacerlo tienen también libertad para ello, siempre que se limiten a reunir con esos objetivos a sus propios militantes.

Sería una verdadera pena si la "alegría" de la plaza se perdiese -como tendería ocurrir si dejase de ser "plaza". Es una alegría -la misma alegría que nos gustaría existiese siempre en "otro mundo posible"- que acaba siendo contagiosa y energizante, alimentada por otro descubrimiento que propicia el Foro, al romper las divisiones que crean compartimentos estancos como consecuencia de las luchas por las transformaciones que crean los diferentes movimientos: la de que somos muchos en la misma lucha. Así que, en el espacio abierto a todos por el Foro, los militantes de los diferentes movimientos se encuentran y se reconocen mutuamente: los que luchan por los derechos de las mujeres, de los trabajadores urbanos y rurales, del medio ambiente, de los niños, los que buscan nuevas relaciones económicas dentro de los países o al nivel de las organizaciones internacionales, los que trabajan por la participación democrática en los gobiernos o por la valoración de la dimensión espiritual del ser humano, etc. etc., en la extensa variedad de los "movimientos" que existen.

Tales militantes de tantas luchas -muchos inclusive largo tiempo separados por opciones ideológicas y partidistas diferentes- encuentran en el Foro una ocasión inédita de conocerse y, quizá, de articularse superando las divisiones que fueron promovidas por los dominadores. Este reencuentro -si así se puede decir- es muchas veces, para muchos, motivo inicialmente de sorpresa, pero enseguida de alegría, ya que se percibieron todos unidos de hecho. 
Aunque el Foro se transformase en un "movimiento de movimientos", ninguno de ellos tendría condiciones para abrir ese espacio y conseguir que todos los demás aceptasen su invitación sin condiciones. El reencuentro sería cercenado por la necesidad de pasar a pertenecer a una estructura que se pretendería unificadora, con todas sus reglas establecidas para ello -acordadas entre todos... Y dentro de ella se instauraría una nueva competencia y con esta una división, como resultado de la lucha por los espacios y por la dirección y definición de los objetivos del nuevo movimiento.

Un último rasgo del carácter del Foro-espacio es el sentimiento de corresponsabilidad que guía la realización de sus eventos. El hecho de ser una plaza sin dueños facilita que esto ocurra así, en mayor medida que en los movimientos en los que se procura desarrollar este sentimiento. En el Foro nadie puede rebelarse contra alguien que se disponga a exigir responsabilidades. Aun los fallos de los organizadores -muchos en general, dada la dimensión que van tomando los eventos-son aceptados y corregidos por la propia iniciativa y creatividad de los participantes. En la edición de 2003 de Porto Alegre, una grave e involuntaria falta -que obligó a los organizadores a multiplicarse para atenuar sus efectos- podría haber hecho perder todo el encuentro: se consiguió publicar el segundo día una programación de los talleres. Los perjudicados encontraron mientras tanto formas de compensar la falta por su propia cuenta, y conseguir incluso iniciativas "externas" -tal como publicación "salvaje" de la programación a partir del momento en que se colocó un "sitio" de todos en Internet, la noche anterior al inicio de los trabajos.

\section{Riesgos que corremos actualmente}

Conservar el FSM como un espacio y por tanto, tal vez, garantizar su mayor riqueza, debe ser resguardado a cualquier precio. Por ello no sería exagerado decir que los que quieren transformarlo en un movimiento acabarán, si lo consiguen, prestando un enorme perjuicio a la causa que nos une a todos -tengan o no clara conciencia de lo que están haciendo, sean movimientos o partidos políticos, y por importantes, estratégicamente urgentes y legítimos que sean sus objetivos. Estarán en verdad actuando contra sí mismos y contra todos nosotros. Estarán obstruyendo y asfixiando su propia fuente de vida -en cuanto aquellas articulaciones e iniciativas nacidas en los propios foros- o al menos desactivando un enorme instrumento de que disponen para expansionarse y para profun- 
dizar su presencia en la lucha en que estamos todos empeñados.

Iniciativas tomadas por un cierto número de movimientos, denominados "movimientos sociales", parecen mientras tanto apuntar en esa dirección. Preocupados con razón por la necesidad de una movilización popular para luchar contra el neoliberalismo, tratan de absorber el Foro para dentro de su dinámica movilizadora y colocarlo al servicio de los objetivos que persiguen.

Tales movimientos saben que no reúnen a todos los participantes de cada evento-aunque congreguen a organizaciones importantes. Pero aun así consideran que su propio documento final podría ser asimilado y presentado como "documento final" del Foro -ya que éste no tiene "documento final"... Una iniciativa en este sentido -nacida en la plaza incubadora del Foro de 2001-ya creó grandes tensiones y malentendidos después de este Foro. Pero las presiones para que ello ocurra se van repitiendo en otros eventos, incluso después del Foro 2003, felizmente con menos desenvoltura, y por poco la última tentativa de hecho no anuló los efectos movilizadores y articuladores que se pretendía obtener con el "Mural de propuestas de acción".

Últimamente una "coordinadora" de estos movimientos ha ido más lejos: participando en los Comités de Organización de los eventos, llevándolos a incluir el último día de la propagación de los mismos una asamblea final que normalmente se realiza al final de los Foros. Esta asamblea, necesariamente parcial, consigue mientras tanto una imagen -por lo menos para los medios de comunicación de masas- de asamblea conclusiva del Foro como un todo. Si esta fórmula pasa a ser adoptada, creará verdaderamente una nueva tensión: la de que cada uno necesitará llevar o hacer llevar a esta asamblea el resultado de la actividad en la que participa, para asegurar que este resultado será implementado por los que a partir de ahí "coordinarán" su efectiva realización, como en un buen y organizado movimiento. Estrechando de esta forma el final del Foro por una asamblea que organizan -y que nunca podrá reunir a todos los que vinieron al mismo- esta asamblea acabará de hecho por ignorar o no respetar las demás propuestas de acción que han surgido. Ello creará la necesidad de "representaciones" que transformarán el Foro en la pirámide de siempre, sin la alegría de la "plaza" horizontal.

Verdaderamente, el gran desafío, en mi opinión, para la continuidad del proceso del Foro y para que éste realice cada vez más plenamente su vocación de incubadora de más movimientos e iniciativas, es el de multiplicar por el resto del mundo tales "espacios" -realmente abiertos y libres, sin 
estrechamientos en torno a propuestas específicas. Esperando que nadie, aun inadvertidamente, contribuya a que el proceso del Foro vaya declinando, hasta desaparecer en cuanto espacio abierto.

Todo esto, sin embargo, es al mismo tiempo una opción a realizar. Las personas y organizaciones que están preparando los eventos de éste y de los próximos años, dentro del proceso del Foro Social Mundial, y los miembros de su actual Consejo Internacional o del Consejo ampliado que se reunirá en junio (2003, nota de la redacción), pueden considerar que deben adoptar una orientación de las preconizadas por los así llamados "movimientos sociales". Nada podría impedirlo. Es una opción. Cada uno de los participantes en el proceso del Foro decidirá entonces sobre la continuidad de su propia participación, incluso porque el Foro no es un movimiento y no tiene reglas sobre la pertenencia al mismo o de respeto a las decisiones mayoritarias tomadas incluso de forma democrática. Lo que no podemos es dejar de discutir de forma clara y franca esta cuestión, para tener plena conciencia de las consecuencias de tales decisiones.

\section{Actividades autogestionadas "versus" programa de los organizadores}

Esta discusión es tanto más necesaria porque, tras las presiones de los participantes para transformar el FSM en un movimiento, los propios organizadores de sus eventos que tienen que adoptar esa opción se resistirán a mantener la actual forma de organización. La opción entre FSM-espacio y FSM-movimiento no puede dejar de repercutir en la organización.

En el Foro-espacio las actividades autoorganizadas tendrían efectivamente prioridad, dentro de la preocupación de los organizadores del evento, puesto que es con ellas como el FSM funciona más claramente como espacio. Lo que se constata en cambio es que se sobrevalora la parte de los eventos programada por los organizadores, en detrimento de los talleres o seminarios programados por los propios participantes. Estas actividades, que constituyen lo esencial de un Foro-espacio, son tratadas casi con desdén. Ellas también parecen secundarias, de menor prestigio e importancia, como si fuesen un peso que los organizadores están obligados a soportar, desde que ese modo de organizar los eventos fue inventado en el Foro de Porto Alegre de 2001.

De hecho, una selección de temas y de oradores en las conferencias o paneles han tomado siempre la mayor parte del tiempo de los organiza- 
dores, en todos los Foros realizados. Ello ocurrió inclusive en el Consejo Internacional: en las reuniones de Bangkok y de Florencia dedicaron gran parte de su programa de trabajo a este tipo de decisiones, para preparar el Foro de Porto Alegre. Se creó una necesidad de reuniones dilatadas fuera de los horarios de reunión del Consejo y hubo incluso una reunión especial en Brasil, entre los encuentros de Bangkok y de Florencia, del nuevo grupo de trabajo que se hizo necesario -de los "coordinadores de los ejes temáticos" con todos los gastos de viaje que tales reuniones acarrean. Verdaderamente los temas y conferenciantes acaban asumiendo un papel de "vitrinas" del Foro, o una demostración pública y visible de lo que se trata en él o de lo que se discute allí, y esto necesita ser montado cuidadosamente, para que queden bien claras sus posiciones y propuestas. Como ocurre también en el Foro de Davos, que no comprende actividades autogestionadas y tiene que seleccionar cuidadosamente, según la coyuntura, el tema central de sus eventos.

Mientras que la preparación de la parte de los eventos programada por sus participantes -que, además de la temática abordada en ella, es una marca diferenciadora del FSM- sigue una dinámica puramente administrativa, casi burocrática: se fija una fecha límite para la inscripción de talleres y seminarios, y se verifica al final del plazo cuáles no pueden ser aceptados, tomando como base la Carta de Principios -análisis que acaba siendo insuficiente, dados los cortos plazos con que se cuenta, disminuyendo en la práctica la negación de inscripción a partidos y organizaciones que declaran explícitamente lo que son... enseguida sigue un trabajo también administrativo de distribución de fechas y locales para esas actividades, y de impresión de un "catálogo" con el nombre de la actividad y su proponente, y una fecha y el local en que se realizará -casi siempre acompañado, además, de las naturales "erratas", al que todos los participantes tienen acceso, con cambios de última hora.

Como, por otra parte, tiende a ser grande el número de estas actividades, solamente algunas mejor distribuidas se realizan en las áreas centrales del evento, y las restantes se distribuyen de la mejor forma posible en el resto del espacio disponibles -a veces en diferentes lugares de las ciudades, muchas veces de difícil acceso. Para completar estas condiciones perjudiciales, el "catálogo" de los talleres o seminarios acaba siendo facilitado en el momento en el que los participantes retiran sus distintivos el primer día del evento -o incluso más tarde, como desgraciadamente sucedió en 2003 en Porto Alegre. 
El resultado de ello es que prácticamente tienden a participar en los talleres y seminarios sus organizadores y los participantes que ellos mismos llegaron a invitar, o que consiguieron determinar a tiempo las actividades que les interesaban.

El cuadro empeora todavía más cuando los organizadores del evento consiguen traer a renombradas figuras para una parte del evento que organizan, y cuando les asignan los mismos horarios de los talleres y seminarios, como ocurrió en Porto Alegre 2003: ya que las grandes conferencias acaban por atraer a la mayoría de los participantes, dejando las actividades autoorganizadas para los que realmente les interesa participar en ellas. Esta perspectiva sobre la función de las grandes conferencias y paneles dentro de un evento debería ser reexaminada.

Varias preocupaciones deberían tomarse para que todo esto no ocurra. Por ejemplo, una fecha limite para la inscripción de talleres y seminarios podría ser fijada un día bastante anterior al inicio del evento -por lo menos dos meses para los grandes Foros. Esto haría posible divulgar las propuestas por Internet con suficiente antelación, permitiendo que se establezcan interrelaciones previas entre los talleres, una distribución de locales y de espacios que facilitara esas interrelaciones, y una mejor distribución de los propios participantes, que ya irían al Foro sabiendo en qué actividades les interesa participar.

Una segunda precaución a adoptar, tal vezigualmente importante, se refiere a los locales de las actividades autoorganizadas: estas deberían celebrarse en el espacio central de los eventos, en la "plaza" principal, con la mejor infraestructura posible, con acceso facilitado y bien divulgado. Durante el tiempo que les estuviese reservado no podrían sufrir la competencia de los eventos destinados al gran público -como ocurrió en 2003 en Porto Alegre, dando la razón a los que llegaron a decir que las grandes "estrellas" usurparon el Foro.

Sin duda una prioridad delas actividades autoorganizadas-que representan en la práctica de la organización de los eventos una opción por el Foro-espacio y no por el Foro-movimiento- sería mucho más favorable para facilitar que se consiguieran efectivamente los objetivos del Foro Social Mundial, formulados en su Carta de Principios e indicados al comienzo de este texto: permitir que el máximo posible de personas, organizaciones y movimientos que se oponen al neoliberalismo puedan encontrarse libremente, escucharse unos a otros, aprender con las experiencias y luchas de los demás, discutir 
propuestas de acción, articularse en nuevas redes y organizaciones que traten de superar el actual proceso de globalización dominado por las grandes corporaciones internacionales y por los intereses financieros. Porque, de hecho, es en los talleres y seminarios autoorganizados donde esto puede ocurrir, y no con los esquemas tradicionales de los grandes encuentros y congresos, en los que las personas escuchan pasivamente lo que las figuras respetables tienden a decirles, pudiendo como máximo, y con mucha suerte, plantearles preguntas.

\section{Comités organizadores: ¿facilitadores o dirección de un movimiento?}

La discusión sobre la opción espacio o movimiento es importante también porque transformar el Foro en un movimiento puede tener efectos negativos para la propia continuidad del proceso, al abrir una posibilidad de disputas por el poder que pueden desgastarlo e incluso destruirlo por dentro. La Carta de Principios del Foro Social Mundial establece que éste no es un espacio de disputa de poder, y su carácter de espacio horizontal y abierto -hasta ahora- ha permitido que efectivamente no haya ocurrido durante sus eventos. Pero la preparación de los eventos no es inmune a ello.

En la medida en que sea visto como movimiento -lo que implica que debe tener una "dirección" política- pasaría a ser estratégico para las fuerzas políticas que participan en él, que traten de integrar sus Comités de Organización, con la perspectiva de influir en sus decisiones. Creándose con ello tensiones entre los que, en cierto sentido, ya se "adueñaron" de él, y los que se sienten "excluidos" o simplemente los que quieren entrar para participar en esa "dirección".

Hay también los que consideran que se debe llevar tal debate al propio Comité de Organización brasileño-actualmente Secretariado del proceso del Foro- o a su Consejo Internacional. Hay que llega a decir que la composición del Comité Brasileño no tiene representatividad, puesto que no cuenta con una participación proporcional de todas las fuerzas o corrientes políticas que deberían estar en la dirección del proceso. O que el Consejo Internacional debería pasar a ser "dirigido" por algunas personas, o "reducido" a un núcleo de "representantes" de los demás.

Estas propuestas tendrían eventualmente razón de ser si el Foro fuese un movimiento, pero no son adecuadas para un Foro-espacio, o una "plaza" que, como ya dijimos anteriormente, no comporta una "dirección política" 
representativa. Ello exige también que todas las personas o entidades dispuestas a prestar el servicio de organizar el uso de la plaza eviten interferir los contenidos que en ellas se discutan y, mucho menos, la libertad que debe estar garantizada a las actividades de los participantes. De cualquier modo, ello depende de personas y organizaciones dispuestas a consagrar su tiempo y sus recursos -como un secretariado ejecutivo- a propiciar el encuentro y la articulación de todos los que están comprometidos con la lucha por "otro mundo".

Parece recomendable que la composición de los Comités de Organización de los Foros-espacios comporte una diversidad que asegure el respeto a la diversidad de los eventos. Pero no será necesario que cuenten con la diversidad y el peso proporcional de las organizaciones y movimientos que participan en los eventos porque esas organizaciones y movimientos no asisten al Foro para recibir directivas. Más importante, sin embargo, que la diversidad en la composición de los Comités es la credibilidad de las personas y organizaciones que los componen. Ellos deben convocar a todos los demás sin que levanten dudas sobre el interés real de esta convocatoria. $\mathrm{O}$ sin que los "convocados" puedan tener el temor de ser instrumentalizados por aquellos que los convocan, para la realización de sus objetivos reales -como puede ocurrir el día en que los partidos políticos decidan asumir "generosamente" el apoyo al proceso.

La perspectiva o concepto que mejor se adapta tanto par los Comités de Organización como para el Consejo Internacional, dentro del Foro-espacio, es la de "facilitador". Los facilitadores no dirigen. Lo que hacen es proporcionar condiciones para que los movimientos que existen o que puedan surgir avancen en sus luchas. Para crear incubadoras de movimientos o de compromisos para construir "plazas" o "fábricas de ideas", no precisan enfrentarse discutiendo alternativas para cambiar el mundo, ni para intentar imponer ideas o propuestas los unos a los otros. Lo que necesitan es preocuparse, dentro de la perspectiva común que adoptan, en hacer que cada evento que organicen cumplan con los objetivos para cuya realización fue creado el Foro. Precisar es escoger y hacer operativo, considerar las coyunturas políticas de cada momento, las mejores alternativas en cuanto al modo de organizar el tiempo y el espacio que será puesto a disposición de los que deben y quieren ir a la plaza para discutir esas alternativas, presentar propuestas de acción, asociarse para realizarlas. 
Naturalmente, otras instancias de valoración y proposiciones sobre el proceso del Foro además de los Comités de Organización de los eventos -comités ampliados, consejos, asambleas- pueden ampliar o afectar al proceso, y conseguirán incorporar más aún una mayor variedad y representación de movimientos y organizaciones comprometidos con la construcción de "otros mundos". Pero, en la opción Foro-espacio, tales instancias -como ocurre con los Comités de Organización-no pueden pretender dirigir tales movimientos y organizaciones, sino solamente dar valor o apoyar la creación de cada vez más espacios-Foros.

Tal perspectiva de trabajo es más difícilmente adoptada porque es mucho menos "heroica" que el ejercicio del liderazgo directamente político, como lo que sería proporcionado por una opción Foro-movimiento, Su adopción incluso puede llevar a una disminución del interés en participar en la organización de los eventos. Se hace más importante reservar fuerzas y recursos para ampliar adhesiones y articulaciones durante el evento...

Pero en el momento en que vivimos es útil y necesario que se rompan las barreras entre distintos tipos y áreas de compromisos; que las articulaciones en la lucha contra el neoliberalismo se extiendan por toda la tierra y se amplíen, se fortalezcan y se acumulen; que nazcan cada vez más movimientos, redes e iniciativas de lucha; que se profundice el debate sobre las propuestas y caminos para superar la dominación del capital; si este es el momento que vivimos, podemos estar seguros de que el servicio de multiplicar los espacios-Foros es inestimable, insustituible y extremadamente meritorio, en la lucha por nuestra causa común. 